\title{
Anomalous Phase Sequences in Lyotropic Liquid Crystals
}

\author{
Won Bo Lee, ${ }^{1}$ Raffaele Mezzenga, ${ }^{2,3}$ and Glenn H Fredrickson ${ }^{1,4,5, *}$ \\ ${ }^{1}$ Department of Chemical Engineering, University of California, Santa Barbara, California 93106, USA \\ ${ }^{2}$ Department of Physics, University of Fribourg, Perolles, Fribourg, CH-1700 Switzerland \\ ${ }^{3}$ Nestlé Research Center, Vers-Chez-Les-Blanc, 1000 Lausanne 26, Switzerland \\ ${ }^{4}$ Materials Research Laboratory, University of California, Santa Barbara, California 93106, USA \\ ${ }^{5}$ Department of Materials, University of California, Santa Barbara, California 93106, USA
}

\begin{abstract}
We present a coarse-grained model in order to describe the unusual sequence of mesophases observed in aqueous solutions of nonionic lipids, such as monoolein. The lipid molecules are modeled as a rigid head and a flexible Gaussian tail, and water is treated explicitly. A key component of the model is thermally reversible hydrogen bonding between the lipid head and water resulting in changes in both head volume and the interactions of the hydrated head with its surroundings. Phase diagrams obtained from unit-cell self-consistent field simulations capture the qualitative thermotropic and lyotropic phase behavior of the monoolein-water system. The unusual phase sequences result from a competition between hydrogen bond formation, changes in head volume and interactions, lipid tail entropy, and the hydrophobic effect.
\end{abstract}

PACS numbers: 61.30.Cz, 64.60.-i, 64.70.Md, 87.15.-v

The mesophases of lipid-water mixtures are of great scientific and technological interest, having relevance to cosmetic, food, and pharmaceutical formulations for the delivery of flavors, drugs, and aromas [1-4]. Cubic bicontinuous phases [5,6], e.g., $I a \overline{3} d$ and $P n \overline{3} m$ and the inverted phases $I a \overline{3} d_{I I}$ and $P n \overline{3} m_{I I}$, are especially useful because they provide pathways for transporting small molecule species such as flavors and nutrients in either aqueous or hydrocarbon phases $[3,4,7]$. While cubic phases are desirable, there is very limited understanding of the relationship between the molecular details of the lipid, its interactions with water, and the presence and placement of cubic phases within the temperature-composition plane. The problem is particularly acute for aqueous solutions of nonionic lipids with a small hydrophilic head, such as the monooleinwater system $[8,9]$. This class of lipid-water mixtures possesses both a normal phase sequence from inverted hexagonal cylinders to inverted cubic gyroid $\left(H_{I I}-I a \overline{3} d_{I I}\right)$ and a reverse phase sequence from lamellar to inverted gyroid $\left(L_{\alpha}-I a \overline{3} d_{I I}\right)$ as the water volume fraction is increased [see Fig. 2(a)]. There is currently no satisfactory theoretical model that explains both the normal and reverse lyotropic phase sequences as well as the changing topology of the phase diagram with temperature. It is this challenge that is addressed in the present Letter.

The concept of a critical packing parameter (CCP) [10], defined as the ratio of the volume of a lipid tail to the product of the cross-sectional area of a lipid head and the length of a lipid molecule, is a useful tool for anticipating membrane curvature and the stability of various lipid and surfactant phases in solution. Nonetheless, the CCP approach predicts only the normal sequence of phases, reflecting a transition in which the water-lipid interfaces are curved less towards the water domains as the concentration of water is increased. The framework does not provide insight into a reverse phase transition such as $L_{\alpha} \rightarrow$ $I a \overline{3} d_{I I}$, where the interfaces go from flat to curved towards water upon increasing the amount of water.

A membrane elasticity model for lipid bilayers initiated by Canham and Helfrich [11,12] invokes phenomenological parameters such as spontaneous curvature, bending rigidity and saddle splay modulus. The model does not elucidate the dependence of these parameters on molecular details of the lipid and solvent, but it can be used to anticipate certain trends in phase behavior [13]. At the other extreme, fully atomistic simulations have been attempted for the monoolein-water system using a classical force field and explicit water [14]. While some promising initial results have been obtained, the time scales of self-assembly in such systems are orders of magnitude beyond those accessible with state-of-the-art computational resources.

Self-consistent field theory (SCFT), which utilizes coarse-grained chain and interaction models, provides a computational strategy intermediate between continuum and atomistic approaches $[15,16]$. It is a powerful and flexible method that has been successfully applied to study the phase behavior of a wide variety of inhomogeneous polymer systems such as block copolymers and polymer alloys [17,18]. Li and Schick [19] have also shown that SCFT models of lipids with flexible Gaussian tails and rigid polar head can predict certain trends in lyotropic behavior. A more detailed SCFT model proposed by Müller and Schick uses the rotational isomeric state (RIS) model to represent lipid tail conformations [20]. Although some trends in experimental data were reproduced, the approach, which did not allow for hydrogen bond formation, did not predict the reverse lyotropic phase sequences that dominate the monoolein-water phase diagram.

In the present Letter, we introduce a novel type of SCFT model that incorporates reversible hydrogen bonding and is 
the first to capture the essential thermotropic and lyotropic physics of lipid-water mixtures in the monoolein class. As shown in Fig. 1(a), monoolein has a monounsaturated lipid tail and a head group that can hydrogen bond with up to ten water molecules. In the coarse-grained description depicted in Fig. 1(b), we simplify the molecule to a flexible Gaussian chain tail consisting of $N$ segments, each of volume $v_{t}$, and a rigid head that can exist in two states: state " 0 ", which has no bound water and a small head volume $v_{h 0}$, and state " 1 ", which has bound water and a larger head volume $v_{h 1}$. The thermally reversible binding of water to the head is a key component of the model, which also influences the head-water and the head-tail interactions. Experimental results on monoolein $[8,9]$ and monolinolein [21] systems suggest that in the bound and hydrated state, the larger state 1 head should interact less favorably with free(unbound) water and lipid tails than a head in the dry state 0 .

Free water in the model is treated simply as a spacefilling pointlike species. Local incompressibility of the solution is imposed and nonbonded interactions among the various species are described by Flory-Huggins $\chi$ parameters. The energy change (in units of the thermal energy $k_{B} T$ ) associated with hydrogen bonding of the head group is denoted by $F_{b}$. Subscripts $h 0, h 1, w$, and $t$ will be used throughout to denote unbound lipid heads, bound lipid heads, free water, and lipid tails, respectively.

Recent work on supramolecular block copolymers [22] has shown that reversible binding can be incorporated in the SCFT framework by working in the grand canonical ensemble where the constraints of chemical reaction equilibria can be imposed on the species activities. Following the same approach here, and using Hubbard-Stratonovich (HS) transforms to transform the grand partition function $\Xi$ into a statistical field theory $[16,17]$, leads to

$$
\Xi \sim \int \mathcal{D} \mathbf{W} \int \mathcal{D} \boldsymbol{\phi} \int \mathcal{D} P e^{-H_{G}[\mathbf{W}, \boldsymbol{\phi}, P]},
$$

where $\boldsymbol{\phi}$ and $\mathbf{W}$ are four-component vector fields representing, respectively, the volume fractions and auxiliary potentials (HS fields) of the $h 0, h 1, w$, and $t$ species. $P$ is a

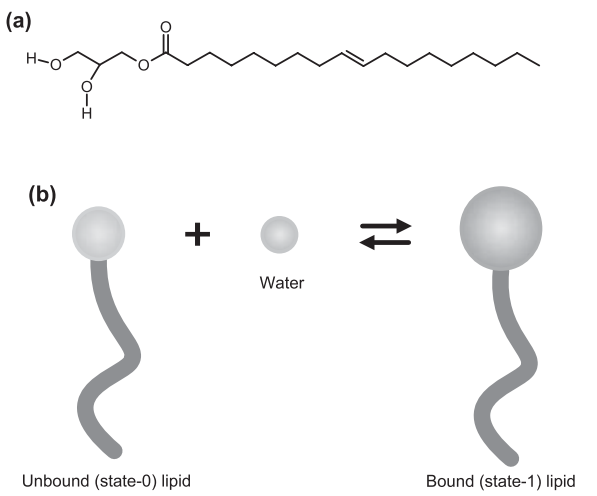

FIG. 1. (a) Chemical structure of a monoolein molecule. (b) Schematic diagram of a lipid-water model. pressure field that imposes the local incompressibility constraint. The effective Hamiltonian appearing in the above field theory can be expressed as

$$
\begin{aligned}
H_{G}= & -Q_{w}-z_{h 0} Q_{h 0}-z_{h 0} \frac{e^{-F_{b}}}{N} Q_{h 1} \\
& +\frac{1}{V} \int d \mathbf{r}\left\{\chi_{h 0, t} N \phi_{h 0} \phi_{t}+\chi_{h 1, t} N \phi_{h 1} \phi_{t}\right. \\
& +\chi_{h 0, w} N \phi_{h 0} \phi_{w}+\chi_{h 1, w} N \phi_{h 1} \phi_{w}+\chi_{t, w} N \phi_{t} \phi_{w} \\
& -\left(W_{h 0} \phi_{h 0}+W_{h 1} \phi_{h 1}+W_{t} \phi_{t}+W_{w} \phi_{w}\right)+P\left(\phi_{h 0}\right. \\
& \left.\left.+\phi_{h 1}+\phi_{t}+\phi_{w}-1\right)\right\},
\end{aligned}
$$

where $V$ is the system volume. Parameters $z_{h 0}$ and $z_{h 1}$ denote the activities of lipids in the unbound and bound states, respectively, in units of $1 / N v_{w}$. Because of incompressibility, only two activities are independent, so $z_{w}$ has been arbitrarily set to 1 . The condition of reaction equilibrium for our two-state model of hydrogen bonding corresponds to [22] $z_{h 1} /\left(z_{w} z_{h 0}\right)=\exp \left(-F_{b}\right) / N$. Nonbonded binary interactions among the relevant $i, j$ species are captured by the Flory-Huggins parameters $\chi_{i, j}$. Finally, the objects $Q_{w}, Q_{h 0}$, and $Q_{h 1}$ are the partition functions of single water molecules, state-0 lipids, and state-1 lipids experiencing fields that are linear combinations of $\mathbf{W}$ and $P$.

In the present Letter, we report only mean-field, or SCFT, solutions of the field theory described in Eq. (1). The mean-field equations result from demanding that $H_{G}$ be stationary with respect to variations of the 9 field components:

$$
\begin{gathered}
\phi_{h 0}=-z_{h 0} \frac{\delta Q_{h 0}}{\delta W_{h 0}}, \\
\phi_{h 1}=-z_{h 0} \frac{e^{-F_{b}}}{N} \frac{\delta Q_{h 1}}{\delta W_{h 1}}, \\
\phi_{t}=-z_{h 0} \frac{\delta Q_{h 0}}{\delta W_{t}}-z_{h 0} \frac{e^{-F_{b}}}{N} \frac{\delta Q_{h 1}}{\delta W_{t}}, \\
\phi_{w}=-\frac{\delta Q_{w}}{\delta W_{w}}, \\
0=\phi_{h 0}+\phi_{h 1}+\phi_{t}+\phi_{w}-1, \\
W_{h 0}=\chi_{h 0, t} N \phi_{t}+\chi_{h 0, w} N \phi_{w}+P, \\
W_{h 1}=\chi_{h 1, t} N \phi_{t}+\chi_{h 1, w} N \phi_{w}+P, \\
W_{t}=\chi_{h 0, t} N \phi_{h 0}+\chi_{h 1, t} N \phi_{h 1}+\chi_{t, w} N \phi_{w}+P, \\
W_{w}=\chi_{h 0, w} N \phi_{h 0}+\chi_{h 1, w} N \phi_{h 1}+\chi_{t, w} N \phi_{t}+P .
\end{gathered}
$$

The functional derivatives in the first four equations can be made explicit through the formulas $[16,18] \phi_{h k}=z_{h k} \frac{v_{h k}}{N v_{w}} \times$ $q_{h k}(\mathbf{r}, 0) q_{h k}^{\dagger}(\mathbf{r}, 1), \quad \phi_{t}=\frac{v_{t}}{v_{w}} \int_{0}^{1} d s \sum_{k=0}^{1} z_{h k} q_{h k}(\mathbf{r}, s) q_{h k}^{\dagger}(\mathbf{r}, 1-$ $s)$, and $\phi_{w}=(1 / N) e^{-W_{w} / N}$, where $q_{h k}$ and $q_{h k}^{\dagger}$ denote 
forward/backward propagators for the tail conformations of a lipid in state $k=0$ or 1 . The forward propagator satisfies the diffusion equation

$$
\frac{\partial q_{h k}(\mathbf{r}, s)}{\partial s}=R_{g}^{2} \nabla^{2} q_{h k}(\mathbf{r}, s)-\frac{v_{t}}{v_{w}} W_{t} q_{h k}(\mathbf{r}, s)
$$

subject to the initial condition $q_{h k}(\mathbf{r}, 0)=e^{-\left(v_{h k} / N v_{w}\right) W_{h k}}$, where $R_{g}=b \sqrt{N} / 6$ is the unperturbed radius of gyration of a lipid tail. The reverse propagator (from the free lipid end) satisfies the same diffusion equation, but with initial condition $q_{h k}^{\dagger}(\mathbf{r}, 0)=1$. The single-molecule partition functions can be similarly expressed: $Q_{h k}=$ $\frac{1}{V} \int d \mathbf{r} e^{-\left(v_{h k} / N v_{w}\right) W_{h k}} q_{h k}^{\dagger}(\mathbf{r}, 1), Q_{w}=\frac{1}{V} \int d \mathbf{r} e^{-\left(W_{w} / N\right)}$.

The SCFT equations, Eqs. (3)-(11) are solved numerically. Initial field configurations are seeded in order to produce a unit cell of a desired mesophase, and the diffusion Eq. (12) is solved pseudospectrally with an operator splitting scheme [23]. Using the calculated propagators, the volume fractions and single-molecule partition functions are evaluated. The $\mathbf{W}$ and $P$ fields are subsequently relaxed using various convergence schemes $[16,24]$ in order to satisfy the remaining SCFT equations, (7)-(11). Simultaneously, $H_{G}$ is minimized with regard to the size and the shape of the unit cell [25]. Phase boundaries are determined by comparing the SCFT free energies among phases at the same value of the activity of a 0 -state lipid, $z_{h 0}$.

Figure 2(b) shows the mean-field phase diagram of our model, where we have assumed a conventional temperature dependance of the tail-water and hydrogen bond interactions, $\chi_{t, w} N=A+B / T,-F_{b}=C / T$, and fixed the bonding to interaction energy ratio $C / B=0.5$. In parameterizing $F_{b}$ this way, we neglect the directionality of the hydrogen bond, which makes an entropic contribution [26] and effectively reduces the bond strength. $A$ is fixed by the choice of a reference set of interaction and bonding parameters, and the other $\chi_{i, j}$ are determined away from the reference point by maintaining constant values of the ratio $\chi_{i, j} / \chi_{t, w}$. The ordinate of Fig. 2(b), $-1 / F_{b}$, can be viewed as a dimensionless temperature, while the abscissa $\phi_{w, \text { tot }}$ is the total volume fraction of water (bound and free). Key to the choice of reference point $\chi$ parameters is a large unfavorable "hydrophobic" interaction between tail and solvent $\chi_{t, w}$, and a head-solvent interaction that is initially small and becomes less favorable upon binding water, $\chi_{h 0, w}<\chi_{h 1, w} \ll \chi_{t, w}$.

The SCFT phase diagram 2(b) captures important qualitative features seen in the experimental diagram, Fig. 2(a). For instance, it provides for a $L_{\alpha} \rightarrow I a \overline{3} d_{I I} \rightarrow H_{I I}$ phase sequence upon increasing temperature. This sequence can be understood by referring to Fig. 3(a), which shows how the composition of the fluid mixture changes along a thermotropic trajectory at $\phi_{w, \text { tot }}=0.175$. As the temperature increases, the volume fraction of bound lipids decreases, and the effective size of the lipid heads also decreases. A smaller lipid head will stabilize mesophases
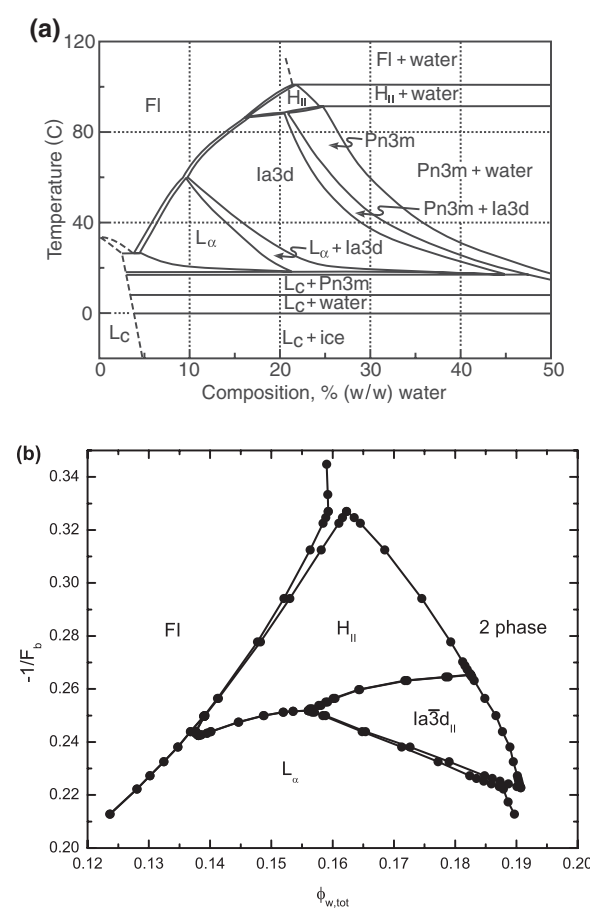

FIG. 2. (a) Experimental phase diagram of the monooleinwater system reprinted from Ref. [9] with permission from Elsevier. (b) Mean-field phase diagram with $v_{h 0} / v_{w}=1.8$, $v_{t} / v_{w}=1.0, N=5$, and $C / B=0.5$. The reference point is $-F_{b}=4.5, \chi_{h 0, t}=1.0, \chi_{h 1, t}=1.45, \chi_{h 0, w}=0.1, \chi_{h 1, w}=0.5$ and $\chi_{t, w}=4.2$. The ratio, $\chi_{i, j} / \chi_{t, w}$, is maintained constant over all values of $-F_{b}$. Labeled phases are $F I$ (lipid-rich homogenous phase), $H_{I I}$ (inverted hexagonal phase), $L_{\alpha}$ (lamellar phase), $I a \overline{3} d_{I I}$ (inverted gyroid phase), and 2 phase (region of coexistence with nearly pure water).

that curve towards water in order to maximize the conformational entropy of the lipid tails.

Figure 2(b) also qualitatively shows the "reverse" lyotropic behavior present in the monoolein-water system. In particular, the $L_{\alpha} \rightarrow I a \overline{3} d_{I I}$ phase transition occurs with increasing water volume fraction. As shown in Fig. 3(b), the $L_{\alpha}$ phase is stable when the bound lipids are saturated and the free water fraction is low, consistent with experimental observations $[9,21]$. However, as more free water is present, the strong hydrophobic interaction between lipid tail and free water increases, which drives a morphological transition to $I a \overline{3} d_{I I}$ wherein the interfacial area between hydrophilic and hydrophobic domains is reduced. The transition is further assisted by an increase in tail entropy [27]. A comparison of the predicted and experimental mesophase lattice constants provides additional support for the model. For example, $d_{I a \overline{3} d_{I I}} / d_{L_{\alpha}} \sim 3.0$ (experiment at $40{ }^{\circ} \mathrm{C}[9,21]$ ) and $\sim 2.8$ (simulation at $-F_{b}=4.2$ ).

We note that the $P n \overline{3} m^{I I}$ (inverted double diamond cubic) phase is always metastable for our parameters, although it most closely competes with $I a \overline{3} d_{I I}$ to the right of the gyroid phase in the mean-field diagram, which is the same sequence as found experimentally. Simulations have 

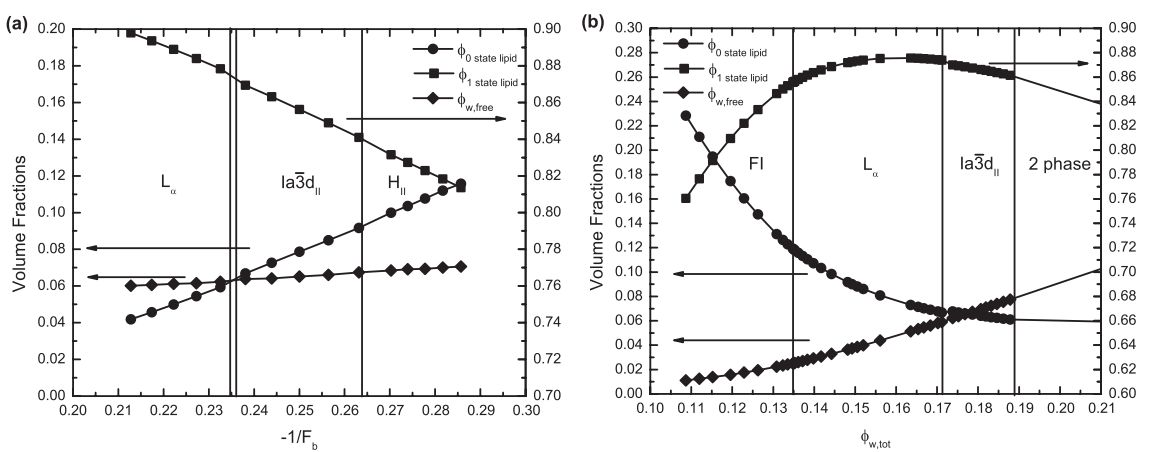

FIG. 3. (a) Species volume fractions versus $-1 / F_{b}$ at fixed $\phi_{w, \text { tot }}=0.175$. (b) Volume fractions versus $\phi_{w, \text { tot }}$ at $-F_{b}=4.2$. also been conducted in which hydrogen bonding was turned off in the model. These studies, which produced only the "normal" phase sequence, verify that head volume changes in response to hydrogen bonding are key to reproducing the lyotropic and thermotropic phase sequences in the monoolein class of systems.

We have conducted some preliminary investigations of the sensitivity of the SCFT phase diagram to the choice of parameters. As $N$ is increased at fixed $v_{t} / v_{w}=1.0$, which corresponds to a smaller water molecule relative to the lipid volume of $N v_{t}+v_{h 0}$, the window of mesophases widens. This is due to an increase in water translational entropy, which favors mesophase swelling. Another important parameter is the ratio of the volume of an unbound lipid head to the volume of a water molecule, $v_{h 0} / v_{w}$. If the value of $v_{h 0} / v_{w}$ is too low, such as $v_{h 0} / v_{w}=1.4$ with $N=6$, the lyotropic $F I$ to $L_{\alpha}$ phase transition disappears, leaving only a $F I$ to $H_{I I}$ transition. The nonbonded hydrophobic interaction parameter between the lipid tail and water, $\chi_{t, w}$, is also key. A large value of $\chi_{t, w}$ gives more stability to the $I a \overline{3} d_{I I}$ phase since it increases hydrophobicity, although it also shifts the mesophases to lower water content. Conversely, if we decrease the value of $\chi_{t, w}$ by more than $6 \%$ at $-F_{b}=4.2$, the $I a \overline{3} d_{I I}$ phase is destabilized relative to $L_{\alpha}$ at all water concentrations. Finally, the hydrated lipid head-water interaction is important to cubic phase stability. When $\chi_{h 1, w}$ is high, the hydrophobic interaction between lipid tail and water is reduced by the bound lipid heads, which favors the $L_{\alpha}$ phase and less swelling. For example, if we increase the value of $\chi_{h 1, w}$ by $20 \%$ at $-F_{b}=4.2$, only the $L_{\alpha}$ phase is stable.

In summary, we have developed a model of nonionic lipid-water mixtures that qualitatively captures the unusual lyotropic and thermotropic phase sequences observed in experiments. Our simulations also represent the first application of self-consistent field theory to inhomogeneous solutions of hydrogen bonding lipids or polymers.

The authors are grateful to K. Katsov and R. Elliott for useful discussions. This work was supported by the MRSEC Program of the National Science Foundation under Grant No. DMR05-20415 and by funding provided to the Complex Fluids Design Consortium at UCSB by the Nestle Research Center. *ghf@mrl.ucsb.edu

[1] S. Walker, M. Kennedy, and J. Zasadzinski, Nature (London) 387, 61 (1997).

[2] I. Koltover, T. Salditt, J. Rädler, and C. Safinya, Science 281, 78 (1998).

[3] D. Discher and A. Eisenberg, Science 297, 967 (2002).

[4] R. Mezzenga, P. Schurtenberger, A. Burbidge, and M. Michel, Nat. Mater. 4, 729 (2005).

[5] V. Luzzati and P. Spegt, Nature (London) 215, 701 (1967).

[6] L. Scriven, Nature (London) 263, 123 (1976).

[7] R. Mezzenga, W. B. Lee, and G. H. Fredrickson, Trends Food Sci. Technol. 17, 220 (2006).

[8] S. Hyde, S. Andersson, B. Ericsson, and K. Larsson, Z. Kristallogr. 168, 213 (1984).

[9] H. Qiu and M. Caffrey, Biomaterials 21, 223 (2000).

[10] J. Israelachvili, Intermolecular and Surface Forces (Academic, New York, 1991), 2nd ed.

[11] P. Canham, J. Theor. Biol. 26, 61 (1970).

[12] W. Helfrich, Z. Naturforsch. C 28, 693 (1973).

[13] U. Schwarz and G. Gompper, Phys. Rev. Lett. 85, 1472 (2000).

[14] S. Marrink and D. P. Tieleman, J. Am. Chem. Soc. 123, 12383 (2001).

[15] M. Matsen and M. Schick, Curr. Opin. Colloid Interface Sci. 1, 329 (1996).

[16] G. H. Fredrickson, The Equilibrium Theory of Inhomogeneous Polymers (Oxford University, New York, 2006).

[17] M. Matsen and M. Schick, Phys. Rev. Lett. 72, 2660 (1994).

[18] M. Matsen, Phys. Rev. Lett. 74, 4225 (1995).

[19] X. Li and M. Schick, Biophys. J. 78, 34 (2000).

[20] M. Müller and M. Schick, Phys. Rev. E 57, 6973 (1998).

[21] R. Mezzenga, C. Meyer, C. Servais, A. Romoscanu, L. Sagalowicz, and R. Hayward, Langmuir 21, 3322 (2005).

[22] E. H. Feng, W. B. Lee, and G. H. Fredrickson, Macromolecules 40, 693 (2007).

[23] G. Tzeremes, K. O. Rasmussen, T. Lookman, and A. Saxena, Phys. Rev. E 65, 041806 (2002).

[24] H. D. Ceniceros and G. H. Fredrickson, Multiscale Model. Simul. 2, 452 (2004).

[25] J. L. Barrat, G. H. Fredrickson, and S. W. Sides, J. Phys. Chem. B 109, 6694 (2005).

[26] H. J. Angerman and G. ten Brinke, Macromolecules 32, 6813 (1999).

[27] V. Kocherbitov, J. Phys. Chem. B 109, 6430 (2005). 